\title{
Escuelas rurales en el Perú: factores que acentúan las brechas digitales en tiempos de pandemia (COVID-19) y recomendaciones para reducirlas
}

\author{
TANIA ANAYA FIGUEROA* \\ Escuela Nacional Superior de Folklore José María Arguedas - Perú
}

JORGE MONTALVO CASTRO $^{* * *}$

Universidad de Lima - Perú

ADOLFO IGNACIO CALDERÓN ${ }^{* * * *}$

Pontificia Universidad Católica de Campinas - Brasil

CLAUDIA ARISPE ALBURQUEQUE ${ }^{* * * *}$

Universidad Privada Norbert Wiener - Perú

Recibido el 05-01-21; evaluado el 10-02-21;

aceptado el 20-02-21

\begin{abstract}
* Este artículo es resultado del curso «Redacción de artículos científicos» realizado por la Escuela de Posgrado de la Universidad Norbert Wiener con apoyo de la Escuela Nacional Superior de Folklore José María Arguedas, el cual fue conducido por el doctor Adolfo Ignacio Calderón, profesor principal de la Pontificia Universidad Católica de Campinas (Brasil), en la condición de profesor visitante. La primera autora expresa especial agradecimiento a la doctora Patricia Medina Zuta, responsable por el curso «Problemática Educativa Nacional» en el Doctorado en Educación de la Universidad San Ignacio de Loyola, el cual sirvió de inspiración para la temática desarrollada en este artículo.

** Magíster en Gestión Pública por la Universidad César Vallejo, con estudios de doctorado en Psicología por la Universidad Nacional Mayor de San Marcos-UNMSM, estudiante del doctorado en Educación de la Universidad San Ignacio de Loyola. Profesora de la Escuela Nacional Superior de Folklore José María Arguedas de Lima. Correo electrónico: hutanafa@hotmail.com. https://orcid.org/0000-0002-5981-918X *** Máster en Tecnologías Integradas y Sociedad del Conocimiento por la Universidad Nacional de Educación a Distancia-UNED (España). Licenciado en Ciencias de la Comunicación por la Universidad de Lima. Miembro del grupo de investigación en Comunicación y Educación del Instituto de Investigación Científica-IDIC de la Universidad de Lima. Correo electrónico: jmontalv@ ulima.edu.pe. https://orcid.org/0000-0001-6404-7981

**** Doctor en Ciencias Sociales por la Pontificia Universidad Católica de San Pablo (Brasil), con posdoctorado en Ciencias de la Educación por la Universidad de Coímbra (Portugal). Profesor principal del Programa de Postgrado en Educación de la Pontificia Universidad Católica de Campinas (Brasil). Investigador de Productividad Científica del Consejo Nacional de Desarrollo Científico y Tecnológico (CNPq) del Brasil. Correo electrónico: adolfo.ignacio@puc-campinas.edu.br. https://orcid. org/0000-0001-6534-2819

***** Doctora en Salud Pública por la Universidad Nacional Federico Villarreal - Perú. Es coordinadora de los programas de Doctorado en Salud y la Maestría de Gestión en Salud de la Escuela de Posgrado de la Universidad Privada Norbert Wiener. Correo electrónico: claudia.arispe@uwiener.edu.pe. https://orcid.org/0000-0003-0792-4655
\end{abstract}




\section{RESUMEN}

Se estudian los factores que acentúan las brechas digitales de las escuelas rurales en el Perú en el contexto de la pandemia del COVID-19. De esa forma, se aborda la educación básica rural antes y durante la pandemia, factores que acentúan las brechas digitales, políticas de inclusión existentes y diversas recomendaciones para reducirlas. Es un estudio cualitativo, analítico-descriptivo, con elementos comparativos entre lo urbano y lo rural, específicamente la región andina del Perú. Las brechas digitales se acentúan por factores de conectividad, dispositivos, apropiación de la tecnología, inadecuada formación docente, entre otros. El estudio evidencia la necesidad de potencializar el binomio conectividad y formación docente para el uso de las tecnologías de la comunicación y de la información y para el diálogo intercultural en los procesos de aprendizaje.

Palabras clave: educación rural, COVID- 19, brecha digital, Programa Aprendo en Casa, escuelas rurales.

\section{Rural schools in Peru: factors that accentuate the digital gaps in times of pandemic (COVID-19) and recommendations to reduce them}

\section{Abstract}

This study points out factors that deepen the digital gaps in rural schools in Peru in the context of the COVID-19 pandemic. Thus, basic rural education is addressed before and during the pandemic a few factors that intensify the digital gaps, existing inclusion policies and several recommendations to reduce them. It is a qualitative, analytical-descriptive study, with comparative elements between urban and rural, specifically in the Andean region of Peru. Some factors accentuate the digital gaps, such as connectivity, devices, appropriation of technology, and inadequate teacher training, among others. The study highlights the need to enhance the binomial connectivity and teacher training for the use of communication and information technologies and for intercultural dialogue in the learning processes.

Keywords: rural education, COVID-19, digital divide, Learning at Home Program, rural schools.

\section{Escolas rurais no Peru: fatores que acentuam as brechas digitais em tempos de pandemia (COVID-19) e recomendaçóes para reduzi-las}

\section{Resumo}

Estudam-se os fatores que acentuam as brechas digitais nas escolas rurais no Peru no contexto da pandemia do COVID-19. Dessa forma, se aborda a educação básica rural antes e durante a pandemia, fatores que acentuam as brechas digitais, políticas públicas existentes e diversas recomendaçóes para reduzi-las. É um estudo qualitativo, analítico-descritivo, com elementos comparativos entre o urbano e o rural, especificamente a região andina do Peru. As brechas digitais se acentuam por 
fatores como conectividade, dispositivos, apropriação da tecnologia, e inadequada formação docente, entre outros. O estudo evidencia a necessidade de potencializar o binômio conectividade y formaçáo docente para o uso das tecnologias da comunicaçáo e da informação e para o diálogo intercultural nos processos de aprendizagem.

Palavras-chave: educação rural, COVID- 19, brecha digital, Programa Aprendo em Casa, escolas rurais.

\section{INTRODUCCIÓN}

A causa de la pandemia por el COVID-19, el Estado peruano dispuso una serie de medidas de emergencia sanitaria, entre ellas el aislamiento social (El Peruano, 2020). En este contexto, a partir de la segunda quincena de marzo de 2020, las escuelas cerraron sus puertas y se dio paso a una educación virtual frente a la suspensión de las clases presenciales, hecho que posibilitó la creación del programa Aprendo en Casa (Ministerio de Educación [Minedu], 2020a) para que la educación básica se desarrolle en la modalidad remota, transmitiéndose en franjas educativas a través de la radio, televisión, plataformas web y redes sociales. Su implementación ha puesto en evidencia la problemática de las escuelas rurales, localizadas en la región de los andes peruanos, referida a una conectividad inadecuada (Prado, 2020), hecho que compromete el aprendizaje de los alumnos, en un contexto en el cual las plataformas digitales dejaron de ser un soporte o auxilio complementario, para convertirse en el principal medio de aprendizaje. Existen dificultades relacionadas con los dispositivos electrónicos, la energía eléctrica, el acceso a medios convencionales (televisión - radio), las condiciones mínimas en el hogar para un proceso de aprendizaje eficaz y la formación de profesores en el uso de las tecnologías de la comunicación y de la información (TIC), y el diálogo intercultural en los procesos de aprendizaje.

Esas dificultades, propias de este siglo, han acentuado las brechas históricamente existentes entre estudiantes del mundo andino y estudiantes que viven en las ciudades, las cuales se extienden desde la época de la Colonia, siglo XVI, cuando primaba una cultura de privilegio económico, político y social, y el indio pasó a trabajar para los espańoles en sus propias tierras, sufriendo marginación y humillación (Gaudin y Pareyón, 2020). Este sistema se mantuvo después de la independencia, durante los siglos XIX y XX, cuando el campesino pasó a ser mano de obra de los grandes hacendados y terratenientes a lo largo y ancho de del Perú (Largo, 2016; Méndez, 2010). 
Dentro de esta realidad, fueron los habitantes de la región andina los que más sufrieron las privaciones y carencias; una situación visibilizada por intelectuales y políticos como Gonzales Prada (Largo, 2016) y José Carlos Mariátegui (Ferrari, 1984), quienes se preocuparon por la problemática del indio. También, desde el ámbito educativo, surgieron propuestas como la de José María Arguedas (Ortiz, 2014), en las que se revaloraba al poblador rural con sus manifestaciones culturales, su propia lengua, el vínculo con la naturaleza y una educación que partía desde el fortalecimiento de su identidad. De acuerdo con Gonzáles (2011), esta propuesta fue complementada por José Antonio Encinas y el proyecto de escuela nueva acorde a la realidad rural, a la que se sumaron Germán Caro Ríos y Walter Peñaloza. La situación en el mundo rural empezó a cambiar después de muchas luchas; sin embargo, las inequidades han continuado a través del tiempo en aspectos como el acceso a los servicios básicos de agua, luz, educación, salud, entre otros.

Según la Comisión Económica para América Latina y el Caribe - Cepal (2020a), en América Latina, el 90\% de los hogares del sector rural no tienen acceso a internet y, de ellos, el grupo etario que tiene menos conectividad son los niños de cinco a doce ańos; asimismo, la velocidad de conexión es tan baja que limita o no permite la educación remota. Ante esta problemática, nos planteamos las siguientes interrogantes: ¿cómo era la escuela rural en el Perú antes de la pandemia?, ¿cómo es la escuela rural en el Perú durante de la pandemia?, ¿cuáles son los factores que acentúan las brechas digitales en la escuela rural?, ¿qué políticas públicas y recomendaciones existen para reducir las brechas digitales en la escuela rural?

Frente a lo expuesto, el presente artículo tiene como objetivo estudiar los factores que acentúan las brechas digitales en las escuelas rurales en el Perú en el contexto de la pandemia del COVID-19, por lo tanto, analiza la educación básica rural antes y durante la pandemia, factores que acentúan las brechas digitales, políticas públicas existentes y diversas recomendaciones para reducirlas.

\section{MarCo teórico}

Conceptualmente, la brecha digital es entendida en este estudio como la desigualdad del acceso a internet y a las tecnologías de la información, infraestructura educativa hoy considerada imprescindible para la educación virtual y para garantizar el derecho al aprendizaje, originada por la inequidad social que ha existido desde siglos atrás generando que la escuela rural tenga un conjunto 
de problemas no resueltos que se agravaron con la llegada de la pandemia del COVID-19.

Las investigaciones sobre las brechas digitales emergen como campo de estudio, en la última década, ganando paulatina visibilidad en importantes y selectivas bases de datos como Scielo (Scientific Electronic Library Online). Por medio del estudio de las brechas digitales se analizan las diferencias de accesibilidad y conectividad a internet que generan desigualdad de oportunidades de desarrollo en la población rural, lo cual está relacionado con la combinación de factores socioeconómicos y tecnológicos (Flores, Hernández y Garay, 2020). En ese campo de estudios se abordan múltiples aspectos de las brechas digitales, tales como las diferencias entre áreas urbanas y rurales o regiones más o menos desarrolladas, problemas de inclusión de personas con discapacidad y necesidades educativas especiales, diferencias de accesibilidad digital entre emprendimientos empresariales y brechas de género en la educación (Mendoza y Caldera, 2014; Souza, Siquiera y Reinhard, 2017; Jiménez y Fernández, 2016).

Este artículo se suma a la literatura científica hispanoamericana, específicamente a la que informa sobre las escuelas rurales, que consensualmente viene defendiendo la «imprescindible» incorporación de las TIC en los procesos educativos de las escuelas rurales "ya que el bienestar y desarrollo social están en juego» (Gilberto, Jurado y Romero, 2014, p. 115), una vez que «se asume que la falta o deficiencia de conocimiento es responsable del retraso por alcanzar el desarrollo, no solo de los individuos sino de la sociedad en general» (Andrade y Campo, 2008, p. 229, citados por Gilberto Hernández, Jurado y Romero, 2014, p. 115). En este sentido, también se abraza el consenso existente en la literatura científica hispanoamericana, en torno del «reconocimiento de que las TIC por sí mismas no mejoran los procesos educativos (Gilberto, Jurado y Romero, 2014, p.115), hecho que también exige otros frentes de actuación, en el ámbito de las políticas educacionales que alcancen el cotidiano escolar, para reducir las brechas digitales.

Los autores del presente artículo están convencidos de que a pesar de que la literatura científica revela la insuficiente producción investigativa en el ámbito académico de Hispanoamérica sobre las TIC en las escuelas y zonas rurales (Gilberto, Jurado y Romero, 2014), la expansión del COVID-19 y la amenaza a la garantía del derecho al aprendizaje de niños y jóvenes en edad escolar exigen esfuerzos redoblados de las diversas comunidades epistémicas para producir conocimiento científico con el intento de descifrar los enigmas impuestos por la pandemia a escala planetaria. 
Todavía son reducidos los trabajos académicos generados en el Perú en el contexto del COVID- 19 sobre las brechas digitales en el campo educativo, hecho que justifica la relevancia de este artículo. Entre los principales trabajos tenemos el estudio de Flores, Hernández y Garay (2020), quienes plantean que se deben generar políticas y medidas económicas para revertir la brecha digital y superar la situación de menor calidad competitiva de los niños y jóvenes. A nivel de Latinoamérica, el trabajo de Nina y Frederico (2020) plantea que la pandemia ha sido un gran desafío para todas las actividades vinculadas a servicios culturales; es así como, en São Paulo, la atención de las bibliotecas ha pasado a ser trabajada mediante redes sociales con gran contenido audiovisual, como cuentacuentos y lectura mediadora. Una investigación en Argentina, Brasil y México aborda los derechos digitales el acceso, la privacidad y la libertad de expresión; y cómo la sociedad civil ha contribuido con la educación remota brindando redes comunitarias de internet (Bizberge y Segura, 2020).

\section{Metodología}

Este artículo es resultado de una investigación de tipo cualitativo, analíticodescriptivo, esencialmente exploratoria frente a una pandemia que, al alterar nuestros hábitos y comportamientos, presenta múltiples interrogantes que desafían a los investigadores de las más diversas áreas del conocimiento. Incluye elementos comparativos entre la realidad de las escuelas urbanas y las escuelas rurales, específicamente de la región de los andes peruanos, con el objetivo de identificar los factores que acentúan las brechas digitales. Los datos recolectados son secundarios, obtenidos por medio de investigación bibliográfica-documental en diferentes fuentes: a) bibliografía referida a la historia de la educación rural, b) artículos científicos contemporáneos presentes principalmente en la base Scielo, sobre las TIC en las escuelas y zonas rurales, brechas digitales en la educación rural y estudios sobre COVID e implicaciones en el área educativa, c) documentos normativos emitidos por organismos internacionales, c) legislaciones y normas oficiales del Ministerio de Educación del Perú, d) estudios producidos por centros de investigación, e) artículos y reportes periodísticos; en estos últimos se ha podido recoger información sobre cómo se viene realizando el programa Aprendo en Casa, especialmente en las escuelas rurales.

Los datos recolectados fueron sistematizados en cuatro tópicos lógicamente concatenados: la educación básica rural antes y durante la pandemia, factores que acentúan las brechas digitales, políticas de inclusión existentes y diversas recomendaciones para reducirlas. 
En términos epistemológicos, los datos fueron interpretados a la luz de la concepción de que la reducción de las brechas digitales en el campo educativo se centran en comprender la inclusión educativa como proceso que visa la garantía del derecho al aprendizaje, teniendo como meta que todos los nińos y jóvenes, independientemente de su condición social, alcancen los objetivos de aprendizaje establecidos por los sistemas educativos nacionales bajo el principio de la equidad escolar, mensurados por los sistemas de evaluación a gran escala (Calderón y Borges, 2020). Esta perspectiva se fundamenta en importantes tesis formuladas, bajo la perspectiva de la garantía del derecho al aprendizaje a lo largo de la vida, en diversos acuerdos internacionales desde la proclamación «Declaración mundial sobre educación para todos: satisfacer las necesidades básicas de aprendizaje», en Jomtien, Tailandia, en 1990 citado en (Calderón Borges, 2020, p. 43). Los esfuerzos por reducir las brechas digitales, en lo que se refiere a las TIC en la educación básica rural, se insertan también en los desafíos impuestos por los Objetivos de Desarrollo Sostenible (ODS), proclamados por la Organización de Naciones Unidas (ONU), planteados con la finalidad de poner fin a la pobreza en el mundo, garantizar la salud, la igualdad de género, la justicia y las oportunidades de aprendizaje durante toda la vida (Cepal, 2016). Este último punto destaca que todo ser humano tiene el derecho a mejorar su calidad de vida y, para ello, los aprendizajes deben ser pertinentes, eficaces, inclusivos y equitativos; de manera que todos puedan tener las mismas oportunidades.

\section{Resultados}

A continuación, se presenta la información obtenida en el estudio:

\subsection{Las escuelas rurales en el Perú antes de la pandemia}

Primero es necesario definir qué se entiende por «rural». Este concepto incluye a todas las personas que habitan y trabajan en el campo, principalmente en actividades agrícolas, ganaderas o extractivas y en el comercio en menor medida. Según Cuesta (2008), el contexto rural encierra una gran complejidad que involucra la diversidad cultural, étnica e idiomática de las comunidades y su dispersión geográfica, además de una gran tradición cultural. El desarrollo de las comunidades rurales en el Perú se ha dado en grados muy diferentes, lo que, en muchos casos, genera una gran desigualdad que se manifiesta en brechas estructurales: ingresos reducidos que no les permiten salir de la pobreza, incapacidad de ahorro e inversión, escasez de infraestructura, limitaciones en educación y salud, diferencias de acceso a servicios e inequidad de género. 
La educación rural se ofrece en territorios y culturas campesinas ubicadas en diferentes regiones del país y que presentan particularidades ambientales, geográficas, históricas y culturales. Las escuelas suelen atender a grupos muy reducidos de estudiantes (Arias, 2017). Tienen diversas modalidades de acuerdo con la cantidad de alumnos, muchas de ellas son multigrados o intercultural bilingüe; es decir, un maestro enseña a más de un grado y a pocos estudiantes, quienes tienen como idioma materno el español, quechua, aimara o una lengua amazónica. Por ello, son los docentes quienes cada año deben elaborar materiales de acuerdo con las necesidades del grupo, ya que los libros entregados por el Minedu casi nunca se adecúan a su realidad. Entonces, el proceso de adecuación del currículo y de todos los recursos pedagógicos es una actividad esencial para el éxito de la educación en el campo.

La educación rural en el Perú ha estado marcada por la historia de la población que vivía en el campo, básicamente dedicada a actividades extractivas como la agricultura, ganadería, pesca, minería, entre otras. Según Méndez (2010) y Morong y Brangier (2019), durante la época de la conquista el delito no era ser conquistador sino indio, al que se le despreciaba por ser proclive al ocio, la holgazanería y la embriaguez, imagen que fue creada y difundida por algunos cronistas. Esta idea se ha venido transmitiendo a través del tiempo $y$, en algunos momentos, ha generado grandes debates entre intelectuales y políticos. Durante la República, con la aparición de las grandes haciendas, los campesinos pasaron a ser la mano de obra del sector más adinerado, pero siempre fueron tratados de manera despectiva, privándolos de acceso a muchos servicios, entre ellos la educación.

Ante la ausencia del Estado, las mismas comunidades campesinas construyeron locales escolares, y buscaban y pagaban a los profesores; lo que empezó a cambiar recién en la década de 1920 (Anaya, 2008). Luego, con la reforma agraria de la década de 1970, la posibilidad de ser dueńos de la tierra les dio una oportunidad para salir de la opresión a la cual estuvieron sujetos durante varios siglos. La construcción de carreteras y su mejoramiento progresivo permitieron la llegada de los medios de transporte y la posibilidad de vincularse con la ciudad. En la actualidad, existen poblaciones rurales que no tienen escuela y otras donde solo hay educación primaria, por lo que los estudiantes de secundaria se deben trasladar a comunidades vecinas caminando varias horas diariamente. Además, la educación pública —a pesar de ser gratuitagenera gastos en los padres, es así como un estudio del año 2000 (Ramírez, 2006) encontró que cada familia gastaba 65 dólares al año por un niño matriculado en educación primaria. 
En el siglo XXI, los gobiernos, las organizaciones regionales y los pobladores del área rural se preocupan por acortar las brechas estructurales mejorando la infraestructura de sus escuelas, comprando equipamiento, capacitando a docentes y adquiriendo libros de trabajo (Gaudin y Pareyón, 2020). Mientras tanto, en las escuelas privadas y los colegios emblemáticos de las grandes ciudades, los estudiantes ya entraban a trabajar incorporando la tecnología digital. Este nuevo recurso educativo implica que ahora la escuela rural tenga un nuevo problema vinculado a la brecha digital en comparación con la escuela urbana.

Los esfuerzos por ampliar el acceso a dispositivos tecnológicos para los estudiantes existen desde hace algunos ańos en algunos países latinoamericanos. Según Katz y Huamán (2014), Chile desarrolló proyectos en torno a los ejes estratégicos de educación, alfabetización digital, red universitaria y gobierno electrónico; y una Agenda Digital basada en la alfabetización digital educativa y comunitaria, el gobierno electrónico y las telecomunicaciones. Dentro de estos proyectos se destacó «Yo elijo mi PC», programa que, hasta 2016, benefició a más de 350000 alumnos. Uruguay también cuenta con proyectos como el plan de Conectividad Educativa de Informática Básica para el Aprendizaje en Línea (Ceibal), que empezó a operar en el año 2007. Su objetivo fue entregar a nińos y maestros computadores portátiles que tienen como características el bajo costo, software libre y resistencia al maltrato de los niños, con la finalidad de disminuir la brecha digital, tanto respecto a otros países como entre los ciudadanos de Uruguay. Una característica importante de este proyecto es que participaron voluntarios (estudiantes, informáticos, ingenieros) en la distribución de las computadoras, la capacitación de los docentes, la instalación de las antenas de internet en las escuelas y hasta en la concepción de herramientas didácticas informáticas (Larrouque, 2017).

En el Perú, con la finalidad de incorporar la tecnología en las escuelas rurales, se creó en 2007, durante el gobierno de Alan García, el programa piloto «Una computadora por niño" para acortar las brechas digitales en las escuelas rurales más pobres. Según una investigación realizada por Ana María Cano en la Universidad de Lima (Quiroz, 2014), se encontró que las competencias de lectura, escritura y matemáticas mejoraron en los niños beneficiados por el proyecto. Sin embargo, las deficiencias fueron la escasa capacitación a los docentes, el temor de los padres a lo nuevo y la ausencia de fluido eléctrico. Al estudiar las barreras en la implantación del referido programa piloto, Laura y Bolívar (2010) destacan a las relacionadas con el profesor y con su capacitación, resaltando: el hecho de que no son nativos digitales, falta de manejo de habilidades tecnológicas básicas, falta de conocimientos para resolver 
problemas técnicos suscitados durante el desarrollo de las sesiones pedagógicas, insuficiente y bajo impacto de las capacitaciones de los profesores, y ausencia de capacitación en estrategias metodológicas con el uso de la portátil y en las necesidades pedagógicas específicas de los profesores

En el año 2016 se realizó una investigación en el Perú con los Niños del Milenio (Cueto y León, 2020), la cual mostró que el 37\% de los jóvenes de quince ańos usaban la computadora y el $56 \%$ accedía a internet diariamente. De igual manera, se encontró que el vínculo con la tecnología estaba en estrecha relación con la educación de la madre, el nivel socioeconómico y la zona de residencia; es así como los jóvenes del área rural tenían menos habilidades digitales que los del ámbito urbano. También se halló que el uso de la tecnología estaba en relación directa con el rendimiento en matemática. Además, se descubrió que, si los niños empezaban a utilizar las computadoras o internet a más temprana edad, sus habilidades digitales eran mayores. En una investigación realizada por Escobar y Gómez (2020) en la región Puno con niños de cuarto año de secundaria, se encontró que el uso de WhatsApp permite desarrollar habilidades comunicativas, logrando mejorar el nivel de comprensión y producción de textos orales y escritos, fomentando el autoaprendizaje de manera entretenida y mejorando la comunicación con el docente.

Según el Instituto Nacional de Estadistica e Informática (INEI, 2017), en el Perú existen 6682 comunidades campesinas, distribuidas en 23 regiones del país. Tumbes es la única región que no registra ninguna comunidad campesina. En Cusco y Ayacucho se encuentra la mayor concentración de estas. El idioma o lengua originaria más hablada es el quechua, en 4601 (68,92\%) comunidades; y el aimara, en $625(9,35 \%)$ centros poblados; mientras que para $1406(21,04 \%)$ comunidades el castellano es su único idioma.

En el aspecto educativo, según el INEI (2017), el 95,2\% de las comunidades cuentan con alguna institución educativa y el 4,8\% no tiene servicios educativos. El 85,8\% tiene servicios de educación inicial, 91,3\% de educación primaria y $40,6 \%$ de secundaria. La población estudiantil rural ascendía a 435437 estudiantes; de ellos, el 51,3\% se encuentra en primaria, 29,2\% en secundaria y $18,6 \%$ en inicial. Respecto a los servicios básicos en las escuelas, el $61,2 \%$ se abastecen de agua por la red pública, el $17,5 \%$ de un pozo y el $20 \%$ no cuentan con este servicio. Asimismo, el 65,5\% tiene alumbrado eléctrico, $4,7 \%$ usa panel solar y $25,5 \%$ no dispone de servicio eléctrico. Estos datos muestran las grandes deficiencias estructurales generadas por factores territoriales, políticos, económicos y socioculturales de la educación rural, lo cual impacta en las brechas digitales y educativas existentes. 


\subsection{Las escuelas rurales en el Perú durante de la pandemia}

Al empezar la pandemia en todo el mundo, los padres han tenido que hacer malabares para que sus hijos realicen sus estudios en los programas implementados por los diversos gobiernos (Unesco, 2020). En el Perú, el sistema "Aprendo en Casa» del Minedu fue diseñado para los colegios públicos. Los programas de radio se difunden en emisoras regionales en espacios de 15 minutos para los primeros grados y 30 minutos para secundaria. En el caso de la televisión, mayormente es por el canal del Estado, con tiempos similares. Ambos medios se complementan con la plataforma web, la cual contiene guía de actividades, recursos y materiales educativos para ser adaptados por los docentes; también incluye recursos para educación intercultural bilingüe y educación primaria multigrado rural.

Según el Minedu (2020a), en el Perú, la matrícula en el área rural para el año 2020 ha sido de 1980133 en la educación básica, 7047 en la educación alternativa y 549 en la educación especial. Una encuesta de este Ministerio (Bustamante, 2020) realizada a padres de familia sobre el medio por el cual acceden al programa "Aprendo en Casa", mostró que el 75\% lo hace por televisión, $18 \%$ por internet y $14 \%$ por radio. La función de los docentes en la implementación del programa ha sido clave, pues han tenido que contactarse por diferentes medios con los padres de familia, usando el teléfono o mensajes de texto en los lugares donde no hay internet; y envío de archivos por WhatsApp para quienes sí tienen acceso.

Para el exministro de Educación, Martín Vegas (2020), «en el ámbito rural, ni la radio, ni la televisión, ni la estrategia digital han funcionado de manera suficiente como para generar un proceso de enseñanza-aprendizaje» (párrafo 3). Las estrategias planteadas tienen limitaciones porque los estudiantes no pueden acceder a la plataforma web o aquellos que acceden lo hacen con una serie de desventajas. Se podría decir que el sistema funciona más como un medio tradicional de educación a distancia vía radio y televisión que como un sistema de plataforma virtual. En el programa «Aprendo en Casa», el trabajo del docente es clave debido al seguimiento que debe realizar a los alumnos, la adaptación de los materiales a la realidad de la escuela y la forma cómo hace llegar a cada estudiante la información. De igual manera, el retorno de las evidencias es muy complicado y la retroalimentación en este contexto casi no existe. En muchos casos, los estudiantes van acumulando contenidos que no comprenden y que, a lo largo del año, van generando retraso o incluso el abandono del sistema escolar. 
Según el Minedu (Gestión, 2020), en el presente año, por efecto de la pandemia, se estima que el Perú tendrá una deserción de 300000 estudiantes, lo que equivale al $15 \%$ de la matrícula nacional. Para enfrentar este problema, el Estado ha implementado, para el sector rural, la entrega de 719000 tabletas con acceso a internet, pero están llegando demasiado tarde. Su distribución recién se ha iniciado a fines del mes de octubre de 2020 y deberá concluir en junio de 2021.

En este contexto, de acuerdo con los estudios del Banco Mundial (Molina, 2020), los estudiantes que no asistan a clases podrían perder de 0,3 a 0,9 ańos, considerando un promedio de 7,7 años de escolaridad; esta pérdida se puede estimar en una disminución de ingresos anuales de 242 a 835 dólares a lo largo de toda la vida, un impacto sujeto al tiempo que las escuelas permanezcan cerradas. El efecto será mayor en los estudiantes que no cuenten en casa con un equipo informático ni acceso a internet. En el caso de las familias rurales, menos del 5\% cuentan con ellos; a esto hay que sumarle que los docentes no se encuentran capacitados para apoyar a los estudiantes. Considerando que "Aprendo en Casa» llega a los hogares a través de radios a pilas o televisor, no es factible que los alumnos repasen las sesiones de clase. También hay que agregar que el horario de transmisión suele coincidir con actividades del hogar en las que los estudiantes apoyan. Por otro lado, según la Encuesta Nacional a Docentes 2018 (Molina, 2020), tres de cada diez profesores de zona rural no cuentan con una computadora ni acceso a internet. Esto implica que existen muchos estudiantes y docentes desconectados.

Otro problema es que los espacios en las casas del área rural a veces son pequeños, debido a que la mayor parte de las actividades familiares se realizan fuera de ella. Además, algunas casas tienen poca iluminación, espacios compartidos con otras actividades y no existe suficiente y adecuado mobiliario; por ejemplo, la mesa que usan los estudiantes normalmente es la que se utiliza para consumir los alimentos (Bustamante, 2020). También encontramos estudiantes que se han hecho cargo de su propio aprendizaje o que uno de mayor edad ayuda al que está en grado inferior; debido a que los padres no están en casa o no están preparados para asumir el rol de mediador en el proceso de enseñanza-aprendizaje. Asimismo, existen niños y jóvenes a quienes sus familias les asignan tareas cotidianas, y terminan descuidando su rol de estudiantes. Finalmente, hay cierta desigualdad entre los niveles educativos; mientras en secundaria los estudiantes muestran más autonomía, en primaria deben ir adquiriendo habilidades de lectoescritura y familiarizarse con los recursos digitales (de existir en su localidad), y los niños de inicial dependen exclusivamente de los adultos. 


\subsection{Factores que acentúan las brechas digitales en las escuelas rurales}

El siglo XXI ha visibilizado grandes diferencias entre los distintos sectores sociales y en 2020, a raíz de la pandemia del COVID-19, la preocupación manifestada desde años atrás por los organizamos internacionales y la comunidad académica hacía prever que el problema de la brecha digital sería uno de los temas de agenda de los gobiernos, y que deberían incorporarlo a sus políticas públicas. Según Bizberge y Segura (2020), la mitad de los pueblos de Latinoamérica no tienen acceso a internet o su conexión es de mala calidad, registrándose mayor desigualdad en las zonas rurales, en las mujeres, niños, jóvenes y adultos mayores.

Según Servindi (2020), solo cuatro de cada diez hogares en las zonas rurales contaban con acceso a internet a fines de 2019. Esta cifra expresa las grandes desigualdades en nivel de ingresos, acceso a la educación y servicios públicos. Sin embargo, el gobierno hace esfuerzos por invertir en infraestructura para mejorar la conectividad regional de banda ancha. Para la Cepal (2020a, 2020b), en Latinoamérica muchos hogares reciben la oferta de conectividad, pero no la contratan debido a que no cuentan con los recursos para pagar los servicios de internet, que son caros y de menor calidad en comparación a los que existen en las ciudades y países desarrollados, por lo que resulta difícil reducir las brechas digitales.

De acuerdo con Venturini (2020) y Bielschowsky y Torres (2018), las brechas digitales están asociadas a diversos factores que es necesario analizar:

a) Conectividad. La baja velocidad de conexión que se da en el área rural $(5,5 \mathrm{Mbb} / \mathrm{s})$ no permite realizar actividades como el teletrabajo y la educación en línea (Cepal, 2020a). Además, la penetración de internet es básicamente a través de la red móvil, siendo cinco veces mayor que la red fija. Las regiones con menor conectividad fija (IPE, 2020) son Pasco $(13,8 \%)$, Ayacucho (13,5\%), Cajamarca $(9,6 \%)$ y Huancavelica $(5 \%)$.

b) Dispositivos. La mayor parte de los estudiantes del área rural usa dispositivos móviles bajo la modalidad de prepago y son modelos de poca capacidad, pues a la hora de comprarlos optan por el dispositivo más barato, lo que no permite descargar y usar varias aplicaciones; si lo hacen el equipo se vuelve muy lento. Además, hay que considerar que estos equipos no fueron diseńados para hacer tareas escolares o leer textos largos.

c) Apropiación de la tecnología. Mientras los niños del área urbana, desde que nacen, viven rodeados de medios electrónicos, los niños del 
área rural viven en contacto con la naturaleza y su acercamiento a la tecnología se da después de tiempo. Muchos de ellos, recién en los últimos años han tenido acceso a equipos digitales, por lo que no se sienten seguros de manejarlos, algo que retarda su aprovechamiento. Sus padres tampoco los pueden ayudar y la labor de entrenarlos debe recaer en personas que sí conocen el dispositivo tecnológico. Se debe considerar también que existen problemas de apropiación de la tecnología por parte de muchos docentes del área rural no solo por la brecha generacional todavía existente, mas también por la carencia de computadoras de uso personal.

d) Precariedad de las viviendas. Algunas casas fueron construidas por los mismos pobladores, sin la orientación profesional de un arquitecto o ingeniero. En ellas, las instalaciones de agua, servicios sanitarios e instalaciones eléctricas se realizaron con posterioridad, de acuerdo con las necesidades que tenían; por lo tanto, la mayoría carece de puntos de carga de energía para los dispositivos electrónicos. También debemos mencionar que todavía existen pueblos donde el fluido eléctrico no se ofrece las 24 horas o donde los pobladores deben ahorrar al máximo para poder pagar los recibos de las empresas prestadoras del servicio.

e) Costo del servicio. Para los pobladores del área rural contratar un servicio fijo o móvil de internet resulta demasiado caro; este puede equivaler al $12 \%$ o $14 \%$ de su ingreso mensual (Cepal, 2020a), por lo que muchos padres de familia no lo pueden adquirir para fines educativos.

Los factores analizados demuestran que las brechas digitales no deben ser concebidas solo como un problema de conectados vs. desconectados, sino desde un enfoque integral de educación y tecnología, con el propósito de enfrentar las desigualdades de origen. Por ello, se debe pensar en un cambio de perspectiva educativa donde todos seamos considerados aprendices: padres, hijos, maestros y estudiantes, para que podamos generar nuevos códigos sociales, culturales, laborales y económicos, y donde el reto sea acortar las brechas digitales (Quiroz, 2014). Para enfrentar este problema se debe, por un lado, lograr que la tecnología llegue a cada uno de los estudiantes de educación básica; por otro, que su uso no solo sea accesible al niño sino a la familia (madre, padre, abuelos) y a todos los pobladores, con la finalidad de que las estrategias de desarrollo local que se implementen en el futuro contemplen el uso de las tecnologías, de manera que se genere progreso en toda la comunidad. 
Por efecto de la pandemia tenemos una escuela rural con una brecha digital muy diversa y heterogénea, pues el impacto está relacionado a diferentes factores socioeconómicos, culturales, familiares e incluso de organización comunal. En aquellos lugares donde los padres tienen acceso a internet, deben adquirir un teléfono para sus hijos gastando entre 100 y 200 dólares por el aparato, y para la conexión deben pagar una tarifa mínima de alrededor de 10 dólares mensuales. En caso de que la municipalidad o comunidad adquiera el servicio, los nińos solo pueden conectarse en sitios cercanos a la antena o punto de acceso; y si no existieran estas condiciones, el profesor debe limitarse a trabajar con la radio, televisión y llamadas telefónicas.

\subsection{Políticas existentes y recomendaciones para reducir las brechas digitales}

En la actual coyuntura educativa, en el Perú, se están acelerando diversas políticas públicas a implementarse para beneficio de las escuelas rurales. Para complementar el programa Aprendo en Casa (Minedu, 2020b), se está entregando un millón de tabletas a los niños de menos recursos. Se trata de una respuesta frente a una situación de emergencia social en tiempos de pandemia, que debería darse permanentemente, desde el momento que el niño ingresa al sistema escolar. También existen otros proyectos a nivel regional y local impulsados desde el sector público y privado, pero son poco conocidos y de impacto limitado.

Considerando que la pandemia ha dejado en el Perú una deserción del 15\% de la matrícula nacional (Gestión, 2020), el Minedu (2020c) ha planteado como estrategias la carpeta de recuperación, la evaluación diagnóstica de entrada en el año 2021, el plan de recuperación, y un periodo de consolidación de competencias entre los meses de marzo a junio. Con estas acciones se intenta evitar la deserción o repitencia de grado de los niños y jóvenes.

Dentro de las políticas del Estado Peruano se encuentran los esfuerzos para impulsar el desarrollo y masificación en todo el territorio nacional de la banda ancha y construcción de la Red Dorsal Nacional de Fibra Óptica (RDNFO), cuyo objetivo es promover el despliegue de infraestructura, servicios, contenidos, aplicaciones y habilidades digitales, como medio que favorece y facilita la inclusión social para lograr la construcción de una sociedad de la información y el conocimiento (Ley 29904, 2015). De acuerdo con documentos oficiales del Ministerio de Transportes y Comunicaciones del Perú (2018), la RDNFO implica el tendido de 13500 kilómetros de fibra en todo el país para brindar internet de alta velocidad y, actualmente, se encuentra desplegado al 100\% en 180 capitales de provincia de las 195 del país. 
La Presidencia de Consejo de Ministros, a partir de un decreto de emergencia, viene implementando el plan «Todos Conectados», que busca contratar servicios de internet satelital con la finalidad de cerrar la brecha digital en la zona rural del país. Dicho plan contempla llegar a 860 comunidades de la selva y 6531 plazas públicas rurales del país (Caretas, 2021). Su implementación se dará durante el año 2021, y se espera que pueda favorecer a miles de estudiantes que el año 2020 no han podido acceder a la educación virtual. También se ve en el horizonte el surgimiento de nuevas posibilidades para ampliar el acceso de las escuelas rurales; el proyecto Starlink (La República, 2021) es una iniciativa privada mundial de internet satelital que probablemente llegará al Perú a fines de 2021, y que puede alcanzar zonas geográficas que las operadoras tradicionales no cubren. El referido proyecto contempla un kit de instalación que tiene un costo de 499 dólares y el precio del servicio de USD 99, monto demasiado alto para las comunidades rurales del país, por lo que se requeriría alguna forma de apoyo económico del Estado o de las grandes empresas que operan en la zona.

Todas estas iniciativas gubernamentales deben ser comprendidas como posibilidades dentro de un amplio abanico de recomendaciones realizadas por diversos organismos internacionales y por la comunidad académica-científica. Sin embargo, la implantación de esas políticas es morosa, con histórica falta de voluntad política y no acompaña la urgencia que la garantía de los derechos al aprendizaje requiere. A ese respecto, uno de los consensos de la literatura científica (Gilberto, Jurado y Romero, 2014), respecto de las TIC en las escuelas rurales, se refiere a la necesidad de «una voluntad política real» para el avance de las políticas de inclusión digital:

así como la asignación constante y suficiente de recursos necesarios. También se recomienda disponer un equipo técnico cuyas labores incluyan su seguimiento y evaluación. El compromiso de los directivos de las instituciones escolares rurales para lograr resultados sigue siendo una exigencia decidida, incluso cuando se impulsa la participación de estos sectores en el diseño de las políticas públicas correspondientes (Gilberto, Jurado y Romero, 2014, p.118).

Los efectos devastadores de la pandemia en el área educativa determinaron una serie de recomendaciones por parte de importantes agencias multilaterales que pueden contribuir para reducir la brecha digital en relación con las escuelas rurales. En ese sentido, la Cepal (2020a), recomienda que todos los países debieran incorporar en sus políticas nacionales el costo de una canasta tecnológica básica para los hogares de escasos recursos que incluya una computadora portátil, un teléfono inteligente o una tableta. De forma 
complementaria, en la Agenda Digital para América Latina (Cepal, 2020b), se propone como objetivos prioritarios el impulso al acceso y la conectividad de banda ancha en las zonas remotas y áreas rurales; y desarrollar una infraestructura adecuada para brindar o fortalecer el servicio educativo.

Las recomendaciones para reducir la brecha digital son convergentes en un especial énfasis en el binomio conectividad y formación de profesores, considerándose también la necesidad de renovación curricular. En esa dirección, para el Grupo de Análisis y Desarrollo-Grade (Cueto y León, 2020), los gobiernos deben priorizar la equidad y a los grupos que tienen menos posibilidad de acceso a recursos digitales, con la finalidad de mejorar su calidad de vida. Bajo esta óptica, recomienda reforzar las capacitaciones a los docentes para incrementar sus habilidades digitales, con el objetivo de que apoyen a los estudiantes en el uso de los recursos informáticos.

La literatura científica en Hispanoamérica sobre TIC y escuelas rurales (Gilberto, Jurado y Romero, 2014), a partir del análisis de diversas experiencias y realidades, destaca consensualmente la resistencia de docentes al uso de estas tecnologías, existiendo también escasa e inadecuada formación del profesorado. De este modo, se recomienda necesaria atención para la formación de profesores en lo que se refiere a las nuevas habilidades digitales y a la necesidad de una formación intercultural que recupere, «en la enseñanza y el aprendizaje las diferencias y las peculiaridades culturales propias de las regiones y que permita enfrentar la desigualdad desde el respeto y el diálogo intercultural»(Quiroz, 2014, p. 72). En este sentido, la literatura científica también es consensual al destacar la importancia del diálogo y coparticipación entre profesores y alumnos de los sectores rurales en los procesos de aprendizaje y en las discusiones sobre la dicotomía local-global, recomendándose que «la clave es la construcción de una práctica educativa realmente participativa, que le permita a las poblaciones rurales comprender la cultura del mundo globalizado, sin perder sus saberes ni su herencia cultural» (Gilberto, Jurado y Romero, 2014, p. 117 ).

En términos de recomendaciones para la renovación curricular, la Agenda Digital para América Latina (Cepal, 2020b), propone «la incorporación de habilidades digitales y competencias en ciencia, tecnología, ingeniería y matemáticas en los procesos de enseńanza-aprendizaje», por medio de la «actualización de los contenidos curriculares y el uso de recursos educativos digitales y estándares de competencia docente acordes a las capacidades que demandarán las actividades del futuro» (Cepal, 2020b, p. 5). También, el Programa Horizontes de la Unesco señala que las escuelas que ya tenían una propuesta pedagógica propia han estado en mejores condiciones para adaptar 
los materiales a su realidad local (Vegas, 2020). Por ello, plantea que en el año 2021 se impulse la elaboración de propuestas curriculares en cada escuela, para atender las necesidades de los estudiantes; en las mismas se debe incluir desarrollo socioemocional, módulos de educación técnica en agricultura, arte regional, industria alimentaria y agricultura orgánica. Asimismo, debe generarse material en las lenguas originarias de los niños.

\section{CONClusiones}

El estudio realizado evidencia que la pandemia ha acentuado las brechas estructurales, históricamente existentes desde la época de la colonización espańola, entre estudiantes de las áreas rurales de los andes peruanos y estudiantes que viven en las ciudades. A diferencia de las áreas urbanas, donde existen escuelas con infraestructura que contribuyen para que los estudiantes desarrollen habilidades digitales desde temprana edad, en las áreas rurales las inversiones gubernamentales son realizadas con muchas limitaciones, estando dirigidas todavía para ofrecer infraestructura básica para la escuela tradicional con limitados esfuerzos en el campo de las TIC.

Evidentemente, existe una acumulación de fragilidades que se acentuaron con la transposición del sistema presencial de enseñanza para la educación virtual como consecuencia de la pandemia. En ese nuevo contexto, las TIC dejaron de ser actores coadyuvantes, como herramientas auxiliares en los procesos de aprendizaje, para ganar la centralidad y el protagonismo, como medio principal para viabilizar los procesos de aprendizaje.

Durante la pandemia, la cobertura educativa de lo que debería ser una auténtica educación virtual, mediada por las plataformas web, asumió la estructura de atendimiento de emergencia, tradicional, de educación a distancia, típico de los movimientos de alfabetización de la década de 1970, teniendo a la televisión y a la radio, con todas sus limitaciones, como principal medio de acceso, debido al atraso existente en las últimas décadas, en la implementación de sólidas bases de conectividad por medio de internet de banda ancha. La carencia de una infraestructura educativa digital determinó la conectividad por medio de herramientas poco adecuadas como teléfono y mensajes de texto frente a la inexistencia de internet fija, principalmente en las áreas rurales.

El retraso en el aprendizaje ocasionado por la pandemia y la ausencia de sólidas políticas de inclusión digital se traduce concretamente en las elevadas tasas de abandono del sistema escolar, en la perdida de ańos de escolaridad y en la reducción de ingresos anuales a lo largo de la vida. Esta realidad adquiere tonos de dramaticidad cuando se verifica que los efectos serán mayores en los 
estudiantes de las áreas rurales, ya que menos de 5\% de las familias cuentan en casa con computadores y acceso a internet. El efecto perverso de las brechas digitales es la reducción de la competitividad en el mercado laboral entre personas del área urbana y el área rural debido a la ausencia de igualdad de oportunidades para todos los estudiantes peruanos.

Las brechas digitales entre estudiantes de escuelas urbanas y rurales están asociadas a diversos factores que las acentúan: la baja velocidad de conexión y la limitada presencia de red fija en las áreas rurales; el uso predominante por parte los estudiantes de dispositivos móviles no adecuados bajo la modalidad de prepago, con modelos de poca capacidad; la reducción de la apropiación de la tecnología por parte de los estudiantes de las áreas urbanas desde la temprana de edad y también de muchos profesores, evidenciándose que existen carencias de computadoras, no solamente por parte de los estudiantes, sino también de los profesores de las áreas rurales; elevados costos de los servicios fijo o móvil de internet.

El Estado peruano viene realizando esfuerzos para impulsar la masificación en todo el país del uso de la banda ancha y ampliar la conexión a los servicios de internet; sin embargo, su implementación se hace morosa frente a su urgencia para la inclusión digital de los estudiantes. La literatura científica es consensual al destacar la necesidad de estructurar sólidas y articuladas políticas en las TIC, con voluntad política para su implementación, participación de los envueltos, asignación suficiente de recursos necesarios y equipos técnicos competentes en el monitoreo y la evaluación.

Para la reducción de la brecha digital, el estudio realizado recomienda la necesidad de potencializar el binomio conectividad y formación docente para el uso de las TIC, y para el diálogo intercultural en los procesos de aprendizaje, una vez que haya un consenso en la literatura científica de que las TIC por sí mismas no mejoran los procesos educativos, sin dejar de lado otras importantes recomendaciones en el campo da renovación curricular para el desarrollo de habilidades socioemocionales, de competencias en ciencias, matemáticas, entre otras áreas e formulación de propuestas curriculares pertinentes.

En términos de conectividad se destacan las recomendaciones de la Cepal (2020a) tales como la implementación de la canasta tecnológica que incluye una computadora portátil, un teléfono inteligente o una tableta; el impulso al acceso y la conectividad de banda ancha en las zonas remotas y áreas rurales; y la necesidad de infraestructura adecuada para ofrecer el servicio educativo.

En términos de formación docente, la Cepal (2020b) destaca el uso de recursos educativos digitales y definición de los estándares de competencia docente. La literatura científica en Hispanoamérica sobre TIC y escuelas 
rurales revaloriza la necesaria atención a la formación de profesores en lo que se refiere a las nuevas habilidades digitales, para su propia práctica docente y para el apoyo a sus estudiantes, y a la necesidad de una formación intercultural en el campo de la enseńanza y aprendizaje para que las poblaciones rurales comprendan el mundo globalizado sin perder sus saberes ni su herencia cultural.

Finalmente, este estudio defiende que alcanzar los objetivos de aprendizaje establecidos por los sistemas educativos nacionales, monitoreados por los sistemas de evaluación a gran escala, como son los exámenes censales, es el gran desafío de este siglo en el ámbito planetario y los gobiernos deben, sin duda alguna, priorizarlos. Reducir las brechas digitales en las escuelas rurales, acentuadas durante la pandemia, es una tarea urgente para permitir a todos los estudiantes peruanos la igualdad de oportunidades y corregir siglos de exclusión y abandono.

\section{REFERENCIAS BIBLIOGRÁFICAS}

Anaya, A. (2008). Santa Catalina de los Baños. Lima: Fargraf.

Arias, J. (2017). Problemas y retos de la educación rural colombiana. Educación y Ciudad, (33), 53-62.https://doi.org/10.36737/01230425.v0.n33.2017.1647

Bielschowsky, R. y Torres, M. (2018). Desarrollo eigualdad: el pensamiento de la CEPAL en su sétimo decenio. Santiago: Cepal. https://doi.org/10.18356/7a7b820c-es

Bizberge, A. y Segura, M. (2020). Los derechos digitales durante la pandemia COVID-19 en Argentina, Brasil y México. Revista de Comunicación, 19(2), 61-85. https://doi.org/10.26441/RC19.2-2020-A4

Bustamante, R. (junio de 2020). Educación en cuarentena: cuando la emergencia se vuelve permanente (segunda parte). GRADE, Grupo de Anàlisis para el desarollo, (5), 1-12. Recuperado de http://www.grade.org.pe/creer/archivos/ articulo-5.pdf

Calderón, A-I. y Borges, R. M. (2020). Avaliação em larga escala na Educação Básica: usos e tensôes teórico-epistemológicas. Meta: Avaliação, 12(34), 28-56. https://doi.org/10.22347/2175-2753v12i34.2281

Caretas (3 de febrero de 2021). MTC anuncia plan "Todos Conectados" para reducir brechas de conectividad en el país. Caretas. Recuperado de https:// bit.ly/3tWNHHO

Cepal (2016). Objetivos para el desarrollo sostenible. Recuperado de https://www. cepal.org/es/temas/agenda-2030-desarrollo-sostenible/objetivos-desarrollosostenible-ods 
Cepal (2020a). Universalizar el acceso a las técnologías digitales para enfrentar los efectos del COVID 19. Informe especial COVID $19 \mathrm{~N}^{\circ} 07$. Recuperado de https://bit.ly/3mqytGm

Cepal (2020b). Agenda digital para América Latina y el Caribe eLAC 2022. Séptima Conferencia Ministerial sobre la Sociedad de la Información de América Latina y el Caribe. Recuperado de https://bit.ly/2Woi4aE

Cuesta, O. (2008). Reflexiones sobre la educación rural en el marco de la comunicación-educación. Civilizar. Ciencias Sociales y Humanas, 8(15), 89-102. https://doi.org/10.22518/16578953.741

Cueto, S., León, J. y Felipe, C. (2020). Acceso a dispositivos y habilidades digitales de dos cohortes en el Perú. [Análisis \& Propuestas, 56]. Lima: GRADE; Niños del Milenio.

Decreto Supremo No 044-2020-PCM, del 15 de marzo de 2020. Decreto Supremo que declara Estado de Emergencia Nacional por las graves circunstancias que afectan la vida de la Nación a consecuencia del brote del COVID-19. Recuperado de https://bit.ly/3agzw8J

Escobar F. y Gómez I. (2020). WhatsApp para el desarrollo de habilidades comunicativas orales y escritas en adolescentes peruanos. Comunicar, 28(65), 111-120. https://doi.org/10.3916/C65-2020-10

Ferrari, A. (1984). El concepto de indio y la cuestión racial en el Perú en los «Siete ensayos» de José Carlos Mariátegui. Revista Iberoamericana, 50(127), 395-408. https://doi.org/10.5195/REVIBEROAMER.1984.3908

Flores, J., Hernández, R. y Garay, R. (2020). Tecnologías de información: Acceso a internet y brecha digital en Perú. Revista Venezolana de Gerencia, 25(90). https://doi.org/10.37960/rvg.v25i90.32396

Gaudin, Y. y Pareyón, R. (2020). Brechas estructurales en América Látina y el Caribe: una perspectiva conceptual-metodologica. Cepal. Recuperado de https://bit. ly/389lTG9

Gestión. (22 de setiembre de 2020). Unos 300,000 Escolares peruanos desertan en medio de la pandemia. Gestión. Recuperado de http://bit.ly/3gUiNK2

Gilberto, O., Jurado, H. y Romero, Y. (2014). Análisis de publicaciones hispanoamericanas sobre TIC en escuelas y zonas rurales. Revista Colombiana de Educación, (66), 105-128. Recuperado de http://www.scielo.org.co/pdf/ rcde/n66/n66a05.pdf

Gonzáles, H. (2011). La experiencia pedagógica de José María Arguedas. Pueblo continental, 22(1), 89-92. Recuperado de http://journal.upao.edu.pe/ PuebloContinente/article/view/454

INEI (2017). I Censo de comunidades campesinas 2017. Resultados dedifitivos. Tomo I. Recuperado de https://bit.ly/2KyjGMe 
IPE (2020). INCORE 2020: Una mirada a la competitividad de la regiones. Lima: Instituto Peruano de Economía.

Jiménez, R. y Fernández, C. (2016). La brecha de género en la educación tecnológica. Ensaio: Avaliação e Políticas Públicas em Educação, 24(92), 743-771. https://doi.org/10.1590/S0104-403620160003000010

Katz, R. y Huamán, C. (2014). Propuesta de fortalecimiento de la gestión de las tecnologias de la información y comunicación (TIC) para impulsar el desarrollo de las TIC en el Perú. Lima: Consejo Nacional de Competitividad Recuperado de http://bit.ly/3rdXEiG

Largo, V. (2016). El problema del indigena en Manuel González Prada. Ogigia. Revista Electrónica de estudios hispánicos, (19), 41-56. https://doi.org/10.24197/ ogigia.19.2016.41-56

Larrouque, D. (2017). Institucionalizar las políticas de inclusión digital: los programas de Argentina, Perú y Uruguay en perspectiva comparada. Polis, 16(48), 107-131. https://doi.org/10.4067/S0718-65682017000300107

Laura, C. y Bolívar, E. (2010). Una laptop por niño en escuelas rurales del Perú: un análisis de las barreras y facilitadores. Economía y Sociedad, (76), 31-38. Recuperado de: http://cies.org.pe/sites/default/files/files/articulos/ economiaysociedad/04-laptops_0.pdf

La República. (11 de febrero 2021). Starlink llega a Perú: ya puedes preordenar internet satelital de Elon Musk. Recuperado de http://bit.ly/2N9pEVx

Ley 29904 del 2015. Ley de promoción de la banda ancha y construcción de la red dorsal nacional de fibra óptica. 24 de abril del 2015. Normativa sobre gobierno digital. Recuperado de https:/www.gob.pe/institucion/pcm/ normas-legales/9863-29904

Méndez, C. (2010). Incas sí, indios no: apuntes para el estudio del nacionalismo criollo en el Perú (Documento de trabajo 56, Serie Historia 10). Lima: IEP. Recuperado de http://repositorio.iep.org.pe/handle/IEP/865

Mendoza, J. y Caldera, J. (2014). Umbrales para la determinación de la brecha digital: comparativa entre regiones desarrolladas. Transinformação, 26(2), 125-132. https://doi.org/10.1590/0103-37862014000200002

Minedu (2020b). Matrícula en el sistema educativo 2020. Lima, Perú. Recuperado de http://escale.minedu.gob.pe

Minedu (11 de octubre de 2020c). Orientaciones para la evaluación de competencias de estudiantes de la Educación Básica en el marco de la emergencia sanitaria por la COVID-19. Recuperado de https://bit.ly/37ceFBL

Ministerio de Transportes y Comunicaciones (2018). Red Dorsal Nacional de Fibra Óptica. Recuperado de https://portal.mtc.gob.pe/comunicaciones/ concesiones/red_dorsal/red_dorsal.html 
Molina, O. (23 de agosto de 2020). El otro lado de la pandemia: las pérdidas en educación. El Comercio. Recuperado de http://bit.ly/2Wo9tow

Morong, G. y Brangier, V. (2019). Los Incas como ejemplo de sujeción. El gobierno del Perú y la escritura etnográfica del oidor de Charcas, Juan de Matienzo (1567). Estudios Atacameños: Arqueologia y Antropología, (61), 5-26. http:// dx.doi.org/10.4067/S0718-10432019005000102

Nina, M. y Frederico, A. (2020). El rol de las bibliotecas en tiempos de COVID-19: reflexiones y propuestas. Desde el Sur, 241-262. https://doi.org/10.21142/ DES-1201-2020-0015

Ortiz, C. (2014). José María Arguedas y la educación. Una aproximación. Yuyaykusun, (7), 15-30. Recuperado de https://revistas.urp.edu.pe/index. php/Yuyaykusun/article/view/219

Prado, E. (29 de octubre de 2020). Hacia la transformación digital del país para remontar la crisis. La República . Perú. Recuperado de http://bit.ly/2LKAvEv

Quiroz, V. (2014). Las brechas digitales en las aulas peruanas. Miradas, (12), 66-73. https://doi.org/10.22517/25393812.9370

Ramírez, E. (2006). Estudio sobre la educación para la población rural en el Perú. Recuperado de https://www.mimp.gob.pe/webs/mimp/sispod/pdf/351.pdf

Resolución Ministerial N 160-2020-MINEDU, del 01 de abril de 2020a. Disponen el inicio del año escolar a través de la implementación de la estrategia denominada "Aprendo en casa», a partir del 6 de abril de 2020 y aprueban otras disposiciones. Recuperado de https://cdn.www.gob.pe/uploads/document/ file/574684/disponen-el-inicio-del-ano-escolar-a-traves-de-la-implementaresolucion-ministerial-n-160-2020-minedu-1865282-1.pdf

Servindi (30 de diciembre de 2020). Brecha digital: el futuro de la próxima generación en riesgo. Recuperado de http://bit.ly/2LLjdYa

Souza, C., Siqueira, É. y Reinhard, N. (2017). Digital divide of small and mediumsized enterprises: an analysis of influencing factors using the toe theory. Ram. Revista de Administração Mackenzie, 18(2), 15-48. https://doi.org/ 10.1590/1678-69712017/administracao.v18n2p15-48

Unesco (abril de 2020). Respuesta del ámbito educativo de la Unesco al COVID-19. Recuperado de https://unesdoc.unesco.org/ark:/48223/pf0000373271_spa

Vegas, M. (15 de diciembre de 2020). Lecciones y desafíos hacia el 2021. El Comercio. Recuperado de http://bit.ly/2LAsPEC

Venturini, J. (27 de marzo de 2020). Los límites de la educación a distancia en América Latina. Recuperado de http://bit.ly/3gWJDS8 